University of Windsor

Scholarship at UWindsor

6-10-2015

\title{
A chi-square method for priority derivation in group decision making with incomplete reciprocal preference relations
}

\author{
Yejun Xu \\ Hohai University \\ Lei Chen \\ Hohai University \\ Kevin Li \\ University of Windsor \\ Huimin Wang \\ Hohai University
}

Follow this and additional works at: https://scholar.uwindsor.ca/odettepub

Part of the Business Commons

\section{Recommended Citation}

Xu, Yejun; Chen, Lei; Li, Kevin; and Wang, Huimin. (2015). A chi-square method for priority derivation in group decision making with incomplete reciprocal preference relations. Information Sciences, 306, 166-179.

https://scholar.uwindsor.ca/odettepub/88

This Article is brought to you for free and open access by the Odette School of Business at Scholarship at UWindsor. It has been accepted for inclusion in Odette School of Business Publications by an authorized administrator of Scholarship at UWindsor. For more information, please contact scholarship@uwindsor.ca. 


\title{
A chi-square method for priority derivation in group decision making with incomplete reciprocal preference relations
}

\author{
Yejun $\mathrm{Xu}^{1,2^{*}}$, Lei Chen ${ }^{1,2}$, Kevin W. Li ${ }^{3}$, Huimin Wang ${ }^{1,2}$ \\ ${ }^{1}$ State Key Laboratory of Hydrology-Water Resources and Hydraulic Engineering, Hohai \\ University, Nanjing, 210098, PR China \\ ${ }^{2}$ Research Institute of Management Science, Business School, Hohai University, Nanjing, \\ 211100, PR China \\ ${ }^{3}$ Odette School of Business, University of Windsor, Windsor, Ontario, Canada N9B 3P4
}

\begin{abstract}
This paper proposes a chi-square method (CSM) to obtain a priority vector for group decision making (GDM) problems where decision-makers'(DMs') assessment on alternative is furnished as incomplete reciprocal preference relations with missing values. Relevant theorems and iterative algorithm about CSM are proposed. Satty's consistency ratio concept is adapted to judge whether an incomplete reciprocal preference relation provided by a DM is of acceptable consistency. If its consistency is unacceptable, an algorithm is proposed to repair it until its consistency ratio reaches a satisfactory threshold. The repairing algorithm aims to rectify an inconsistent incomplete reciprocal preference relation to one with acceptable consistency in addition to preserving the initial preference information as much as possible. Finally, four examples are examined to illustrate the applicability and validity of the proposed method, and comparative analyses are provided to show its advantages over existing approaches.
\end{abstract}

Keywords: Group decision making; chi-square method; incomplete reciprocal preference relation; priority; consistency.

\section{Introduction}

Group decision making (GDM) $[9,13,14,18,21,24,35]$ is a procedure of drawing on the combined wisdom and experience of experts from different domains

${ }^{*}$ Corresponding author. Tel. +86-25-68514612;

E-mail address: xuyejohn@163.com(Y.-J.Xu). 
to rank a finite number of alternatives. Reciprocal preference relations $[21,23,27,34$, 39] are commonly used to represent decision-makers (DMs)' preferences over a set of possible alternative solutions, and have received considerable research attention in the past decades. However, owing to time pressure, lack of knowledge, and the DM's limited expertise in the specific problem domain $[1,4,5,33,36,37,41-44]$, sometimes a DM can at best furnish his/her judgment on alternatives as a reciprocal preference relation with missing or incomplete entries. Therefore, the method to derive priorities from incomplete reciprocal preference relations $[3,10,11,15,41]$ has presented itself as an important and promising research topic, and attracted considerable research interest.

For example, $\mathrm{Xu}$ and $\mathrm{Da}[32]$ put forward a normalizing ranking aggregation method (NRAM) to derive priorities from an incomplete reciprocal preference relation. $\mathrm{Xu}$ and Wang [40] extended the well-known eigenvector method (EM) for priority derivation for an incomplete reciprocal preference relation, and the improvement method therein not only increases the consistency level but also preserves the initial preference information as much as possible. It is worth noting that the aforementioned NRAM and EM can only be applied to a single incomplete reciprocal preference relation. $\mathrm{Xu}$ [41] proposed two goal programming models (GPM) to obtain a collective priority vector from several incomplete reciprocal preference relations. Gong [17] put forward a least-square method (LSM) to generate a collective priority vector from incomplete reciprocal preference relations furnished by multiple DMs. Gong's approach results in a simple equation. But it cannot be applied to obtain a priority vector when the matrix $Q$ is singular or $Q^{-1}$ does not exist. In contrast to LSM, which is only applicable to the case with at least one multiplicative inconsistent incomplete reciprocal preference relation, the logarithmic least squares method (LLSM) put forward by Xu et al. [38] can be used for all incomplete reciprocal preference relations regardless of their multiplicative consistency property. In real-world decision processes, different DMs often carry heterogeneous power in reaching the final recommendation. It is noted that the aforementioned methods did not take into account DMs' weights in the decision 
process.

This paper extends a chi-square method (CSM) to prioritize alternatives in a GDM context when DMs furnish their judgment as incomplete reciprocal preference relations. The CSM was initially developed for priorities by Jensen [19], and was later cited by Blankmeyer [7]. The original approach is complicated and has rarely been used. Wang and $\mathrm{Fu}$ [28] developed a convergent and simple iterative algorithm to facilitate its application in practice. Due to its nonlinear property, this improved algorithm has many advantages such as ease in computer implementation. As such, the extended CSM has arisen as a simple but efficient approach to deal with incomplete reciprocal preference relations.

The key motivations to adopt the CSM can be summarized as follows: (1) The CSM can be used to obtain a collective priority vector from several incomplete reciprocal preference relations, while other methods such as EM and NRAM can only be applied to a single incomplete reciprocal preference relation. This advantage makes it a natural choice for handling GDM. (2) The CSM is convenient in considering different DMs' weights in the decision process while this issue has been largely omitted by other methods. (3) By properly setting model parameters, the CSM can be flexibly employed to handle both complete and incomplete reciprocal preference relations. (4) Compared with other methods, the CSM is known for its better fitting performance, rank preservation capability and discrimination power. After Wang and $\mathrm{Fu}[28]$ 's extension, the improved CSM has become an efficient and convenient tool to handle incomplete reciprocal preference relations. By exploiting CSM to derive priority weights from incomplete reciprocal preference relations in a GDM context, this article further enhances its applications and enriches the theory and methodology of priority derivation.

An important issue in GDM with incomplete reciprocal preference relations is consistency test and inconsistency repairing because consistency of the judgment given by DMs has a direct impact on the final decision result [22]. Xu and Wang [40] adapted Saaty [26]'s consistency ratio $(C R)$ to a fuzzy context and introduced a so-called fuzzy consistency ratio $(F C R)$, which can be applied to incomplete 
reciprocal preference relations. By adopting Saaty's suggested threshold, an incomplete reciprocal preference relation is deemed to be acceptably consistent if $F C R<0.1$ [24]. If an incomplete reciprocal preference relation given by the DM does not possess acceptable consistency, it has to be repaired so that its consistency reaches the acceptable threshold. This paper will put forward a CSM-based algorithm to accomplish this task.

The remainder of the paper is organized as follows. Section 2 provides a review on basic concepts of reciprocal preference relations, incomplete reciprocal preference relations and an acceptable FCR. An associated theorem is also presented. In Section 3 , the CSM is extended to obtain a priority vector from incomplete reciprocal preference relations based on the multiplicative transitivity property, resulting in an iterative algorithm. Section 4 puts forward an approach to repair an unacceptably inconsistent incomplete reciprocal preference relation to derive one with acceptable consistency. In Section 5, four examples are examined to show how to apply the proposed CSM and its effectiveness in handling GDM problems. Comparative analyses with existing methods demonstrate its validity and advantages. Concluding remarks are furnished in Section 6.

\section{Preliminaries}

In this section, we will give the definitions of reciprocal preference relations, incomplete reciprocal preference relations and a FCR.

Denote $N=\{1,2, \ldots, n\}, M=\{1,2, \ldots, m\}$. Let $X=\left\{x_{1}, x_{2}, \ldots, x_{n}\right\} \quad(n \geq 2)$ be a finite set of alternatives, where $x_{i}$ denotes the $i^{\text {th }}$ alternative. $E=\left\{e_{1}, \ldots, e_{m}\right\}$ be a finite set of experts, where $e_{k}$ stands for the $k^{\text {th }}$ expert. $H=\left(h_{1}, \ldots, h_{m}\right)^{T}$ be the weight vector of experts, where $\sum_{k=1}^{m} h_{k}=1, h_{k} \geq 0$ and $h_{k}$ demonstrates the importance degree of expert $e_{k}$ in the decision process.

A fuzzy preference relation is defined as follows $[9,16,44]$. The preference information on $X$ is described by a fuzzy preference relation $R \subset X \times X$, 
$R=\left(r_{i j}\right)_{n \times n}$, with membership function $\mu_{R}: X \times X \rightarrow[0,1]$, where $\mu_{R}\left(x_{i}, x_{j}\right)=r_{i j}$ denotes the preference degree of alternative $x_{i}$ over $x_{j} . r_{i j}=0.5$ indicates the DM's indifference between $x_{i}$ and $x_{j}, r_{i j}=1$ signifies that $x_{i}$ is definitely preferred to $x_{j} .0 \leq r_{i j}<0.5$ implies that $x_{j}$ is preferred to $x_{i}$ and the smaller $r_{i j}$ the stronger the preference of alternative $x_{j}$ over $x_{i} .0 .5<r_{i j}<1$ means that $x_{i}$ is preferred to $x_{j}$ and the greater $r_{i j}$ the stronger the preference of alternative $x_{i}$ over $x_{j}$

Definition 1 [21]. Let $R=\left(r_{i j}\right)_{n \times n}$ be a fuzzy preference relation, then $R$ is called a reciprocal preference relation if

$$
r_{i j} \in[0,1], \quad r_{i j}+r_{j i}=1, \quad r_{i i}=0.5, \text { for all } i, j \in N .
$$

Definition $2[12,27]$. Let $R=\left(r_{i j}\right)_{n \times n}$ be a reciprocal preference relation, then $R$ has multiplicative transitivity property if

$$
\left(\frac{1}{r_{i j}}-1\right)\left(\frac{1}{r_{j k}}-1\right)=\frac{1}{r_{i k}}-1, i, j, k \in N .
$$

It has been found [17] that a perfectly multiplicative transitivity reciprocal preference relation $R=\left(r_{i j}\right)_{n \times n}$ can be precisely characterized by a priority vector $W=\left(w_{1}, w_{2}, \ldots, w_{n}\right)^{T}, \quad$ where $\quad r_{i j}=w_{i} /\left(w_{i}+w_{j}\right), \quad \sum_{i=1}^{n} w_{i}=1 \quad$ and $\quad w_{i}>0$ for $i=1,2, \ldots, n$.

Definition 3 [2]. A membership function $f: X \rightarrow Y$ is called partial if at least one element in the set $X$ is not mapped to an element in the set $Y$. If every element from the set $X$ is mapped to an element in $Y$, then we have a total function.

Definition 4 [2]. A reciprocal preference relation $P$ on a set of alternatives $X$ with a partial membership function is an incomplete reciprocal preference relation.

For any $i, j \in N$, let $c_{i j}$ be the $i j^{\text {th }}$ entry of an incomplete preference relation 
$139 C=\left(c_{i j}\right)_{n \times n}, \quad \delta_{i j}=\left\{\begin{array}{ll}1 & c_{i j} \neq- \\ 0 & c_{i j}=-\end{array}\right.$, and $c_{i j}=-$ indicates a missing element $c_{i j}$.

140 According to Definition 3, $\delta_{i j}=1$ if and only if there exists $c_{i j}=\mu_{C}\left(x_{i}, x_{j}\right), \delta_{i j}=0$

141 denotes that the preference value $c_{i j}=\mu_{C}\left(x_{i}, x_{j}\right)$ is not furnished or missing.

142 Theorem 1 [20]. Let $C=\left(c_{i j}\right)_{n \times n}$ be an incomplete reciprocal preference relation,

143 Then $C$ can be completed by the known elements if there exists at least one known 144 non-diagonal element in each row or column of $C$. This implies that an incomplete 145 reciprocal preference relation $C$ which can be completed has at least (n-1) 146 non-diagonal judgments.

147 Let $C=\left(c_{i j}\right)_{n \times n}$ be an incomplete reciprocal preference relation, its fuzzy 148 consistency index and fuzzy consistency ratio are denoted by FCI and FCR for short, 149 and their formulas are presented as follows [38].

$$
\left\{\begin{array}{l}
F C I=\frac{1}{n(n-1)} \sum_{1 \leq i<j \leq n} \sigma_{i j} \delta_{i j}\left(\frac{c_{i j}}{c_{j i}} \frac{w_{j}}{w_{i}}+\frac{c_{j i}}{c_{i j}} \frac{w_{i}}{w_{j}}-2\right) \\
F C R=\frac{F C I}{R I}
\end{array}\right.
$$

where $\sigma_{i j}$ and $\delta_{i j}$ are binary variables defined below and $R I$ is the mean

consistency index of randomly generated multiplicative preference matrices as given in Table 1.

$$
\begin{aligned}
\sigma_{i j} & =\left\{\begin{array}{ll}
0, & \text { if } c_{i j}=0 \text { or } 1, \\
1, & \text { otherwise. }
\end{array}, \quad i, j \in N .\right. \\
\delta_{i j} & =\left\{\begin{array}{ll}
0, & \text { if } c_{i j}=-, \\
1, & \text { if } c_{i j} \neq-,
\end{array}, i, j \in N .\right.
\end{aligned}
$$
Definition 5 [38]. Let $C=\left(c_{i j}\right)_{n \times n}$ be an incomplete reciprocal preference relation, if $F C R<0.1$, then $C$ is of acceptable consistency, otherwise $C$ 's consistency level is unacceptable.

160 Table 1. The mean consistency index of randomly generated matrix [26]

\begin{tabular}{ccccccccccccccll}
\hline$n$ & 1 & 2 & 3 & 4 & 5 & 6 & 7 & 8 & 9 & 10 & 11 & 12 & 13 & 14 & 15 \\
\hline$R I$ & 0 & 0 & 0.52 & 0.89 & 1.12 & 1.26 & 1.36 & 1.41 & 1.46 & 1.49 & 1.52 & 1.54 & 1.56 & 1.58 & 1.59 \\
\hline
\end{tabular}




\section{A chi-square method for priority derivation from group incomplete reciprocal preference relations}

Consider a GDM problem, where $m$ DMs give their preferences in the form of reciprocal preference relations, i.e. expert $e_{k}$ describes his/her preference information as $R^{(k)}=\left(r_{i j}^{(k)}\right)_{n \times n}$.

Let $W=\left(w_{1}, w_{2}, \ldots, w_{n}\right)^{T}$ be the priority weight vector for the reciprocal preference relations $R^{(k)}=\left(r_{i j}^{(k)}\right)_{n \times n}$, where $\sum_{i=1}^{n} w_{i}=1, \quad w_{i}>0, \quad i \in N$. If $R^{(k)}=\left(r_{i j}^{(k)}\right)_{n \times n}$ is a complete reciprocal preference relation with multiplicative transitivity then it can be expessed as [17]

$$
r_{i j}^{(k)}=\frac{w_{i}}{w_{i}+w_{j}}, \quad i, j \in N
$$

If some elements of $R^{(k)}$ are missing or not given by the DM, then $R^{(k)}$ is an incomplete reciprocal preference relation. We shall extend Eq. (4) to the case of incomplete reciprocal preference relations. For computational convenience, an indicator matrix $\Delta=\left(\delta_{i j}\right)_{n \times n}$ is constructed for incomplete reciprocal preference relation $C^{(k)}=\left(c_{i j}^{(k)}\right)_{n \times n}$ as follows

$$
\delta_{i j}^{(k)} c_{i j}^{(k)}=\delta_{i j}^{(k)} \frac{w_{i}}{w_{i}+w_{j}}, i, j \in N
$$

where $\delta_{i j}^{(k)}$ is a binary variable defined as [41]:

$$
\delta_{i j}^{(k)}= \begin{cases}0, & c_{i j}^{(k)}=- \\ 1, & c_{i j}^{(k)} \neq-.\end{cases}
$$

Due to additive reciprocity, it is easy to find that $\delta_{i j}^{(k)}=\delta_{j i}^{(k)}$.

Next, we turn to find a priority vector $W=\left(w_{1}, w_{2}, \ldots w_{n}\right)^{T}$ to satisfy Eq.(5), where $\sum_{i=1}^{n} w_{i}=1, w_{i} \geq 0$. To accomplish this, the following chi-square optimization model is constructed: 


$$
\begin{aligned}
& \text { s.t. }\left\{\begin{array}{l}
\sum_{i=1}^{n} w_{i}=1, \\
w_{i}>0, \quad i \in N .
\end{array}\right. \\
& D_{W}=\left\{W=\left(w_{1}, w_{2}, \ldots, w_{n}\right)^{T} \mid \sum_{i=1}^{n} w_{i}=1, w_{i}>0, i \in N\right\} .
\end{aligned}
$$
chi-square model, the following theorem is established.

Theorem 2. $F(W)$ has a unique minimum point $W^{*}=\left(w_{1}, w_{2}, \ldots w_{n}\right)^{T} \in D_{w}$, which is also the unique solution of the following system of equations in $D_{W}$ :

$$
\sum_{j=1}^{n} \sum_{k=1}^{m} h_{k} \delta_{j i}^{(k)} c_{j i}^{2(k)} \frac{w_{i}}{w_{j}}=\sum_{j=1}^{n} \sum_{k=1}^{m} h_{k} \delta_{i j}^{(k)} c_{i j}^{2(k)} \frac{w_{j}}{w_{i}}
$$

Proof. As $D_{W}$ is a bounded vector space and $F(W)$ is continuous function in $D_{W}$, for $F(W) \geq 0, w \in D_{w}, F(W)$ therefore has an infimum, namely there exists $w \in D_{w}$ such that function $F(W)$ reaches its minimum value. following Lagrangian function is constructed.

$$
L(W, \lambda)=F(W)+\lambda\left(\sum_{i=1}^{n} w_{i}-1\right) .
$$
$w_{i}$ to be zero, we obtain the following set of equations: 


$$
\frac{1}{w_{i}} \sum_{j=1}^{n} \sum_{k=1}^{m} h_{k}\left(\delta_{j i}^{(k)} c_{j i}^{2(k)} \frac{w_{i}}{w_{j}}-\delta_{i j}^{(k)} c_{i j}^{2(k)} \frac{w_{j}}{w_{i}}\right)+\lambda=0, \quad i \in N
$$

204

which is equivalent to

$$
\sum_{j=1}^{n} \sum_{k=1}^{m} h_{k}\left(\delta_{j i}^{(k)} c_{j i}^{2(k)} \frac{w_{i}}{w_{j}}-\delta_{i j}^{(k)} c_{i j}^{2(k)} \frac{w_{j}}{w_{i}}\right)+\lambda w_{i}=0, \quad i \in N
$$

Summing up Eq. (14) with respect to $w_{i}, i \in N$, we have

$$
\sum_{i=1}^{n} \sum_{j=1}^{n} \sum_{k=1}^{m} h_{k}\left(\delta_{j i}^{(k)} c_{j i}^{2(k)} \frac{w_{i}}{w_{j}}-\delta_{i j}^{(k)} c_{i j}^{2(k)} \frac{w_{j}}{w_{i}}\right)+\lambda \sum_{i=1}^{n} w_{i}=0
$$

Since $\sum_{i=1}^{n} \sum_{j=1}^{n} \sum_{k=1}^{m} h_{k}\left(\delta_{j i}^{(k)} c_{j i}^{2(k)} \frac{w_{i}}{w_{j}}-\delta_{i j}^{(k)} c_{i j}^{2(k)} \frac{w_{j}}{w_{i}}\right) \equiv 0$ and $\sum_{i=1}^{n} w_{i}=1$, we have $\lambda=0$. Plugging $\lambda=0$ into Eq. (14), one has

$$
\sum_{j=1}^{n} \sum_{k=1}^{m} h_{k}\left(\delta_{j i}^{(k)} c_{j i}^{2(k)} \frac{w_{i}}{w_{j}}-\delta_{i j}^{(k)} c_{i j}^{2(k)} \frac{w_{j}}{w_{i}}\right)=0, \quad i \in N
$$

That is

$$
\sum_{j=1}^{n} \sum_{k=1}^{m} h_{k} \delta_{j i}^{(k)} c_{j i}^{2(k)} \frac{w_{i}}{w_{j}}=\sum_{j=1}^{n} \sum_{k=1}^{m} h_{k} \delta_{i j}^{(k)} c_{i j}^{2(k)} \frac{w_{j}}{w_{i}}, \quad i \in N
$$

It is clear that the minimum point $W^{*}$ is a solution to Eq. (10), if the solution is unique in $D_{w}, W^{*}$ can be uniquely determined. The uniqueness is proved by contradiction as follows.

Assume that $V=\left(v_{1}, v_{2}, \ldots, v_{n}\right)^{T} \in D_{w}$ and $W=\left(w_{1}, w_{2}, \ldots, w_{n}\right)^{T} \in D_{w}$ are two solutions to Eq. (10). Let $u_{i}=w_{i} / v_{i}, i \in N$ and $u_{l}=\max _{i \in N}\left\{u_{i}\right\}$. If there exists $j \in N$ such that $u_{j}<u_{l}$, then we have

$$
\begin{aligned}
& \sum_{j=1}^{n} \sum_{k=1}^{m} h_{k} \delta_{l j}^{(k)} c_{l j}^{2(k)} \frac{v_{j}}{v_{l}}>\sum_{j=1}^{n} \sum_{k=1}^{m} h_{k} \delta_{l j}^{(k)} c_{l j}^{2(k)} \frac{v_{j}}{v_{l}} \cdot \frac{u_{j}}{u_{l}}=\sum_{j=1}^{n} \sum_{k=1}^{m} h_{k} \delta_{l j}^{(k)} c_{l j}^{2(k)} \frac{w_{j}}{w_{l}} \\
& \sum_{j=1}^{n} \sum_{k=1}^{m} h_{k} \delta_{j l}^{(k)} c_{j l}^{2(k)} \frac{v_{l}}{v_{j}}<\sum_{j=1}^{n} \sum_{k=1}^{m} h_{k} \delta_{j l}^{(k)} c_{j l}^{2(k)} \frac{v_{l}}{v_{j}} \cdot \frac{u_{l}}{u_{j}}=\sum_{j=1}^{n} \sum_{k=1}^{m} h_{k} \delta_{j l}^{(k)} c_{j l}^{2(k)} \frac{w_{l}}{w_{j}}
\end{aligned}
$$

According to Eqs. (10), (18), (19), it can be deduced that 
$221 \quad \sum_{j=1}^{n} \sum_{k=1}^{m} h_{k} \delta_{l j}^{(k)} c_{l j}^{2(k)} \frac{w_{j}}{w_{l}}<\sum_{j=1}^{n} \sum_{k=1}^{m} h_{k} \delta_{j l}^{(k)} c_{j l}^{2(k)} \frac{w_{l}}{w_{j}}$

222 which contradicts Eq. (10), Thus, $u_{j}<u_{l}$ cannot hold. Therefore, for all $j \in N$,

$223 u_{j}=u_{l}$, namely, $w_{1} / v_{1}=w_{2} / v_{2}=\ldots=w_{n} / v_{n}$. Due to the fact that $\sum_{i=1}^{n} v_{i}=1$,

$224 \sum_{i=1}^{n} w_{i}=1$, we have $w_{i} \equiv v_{i}, \forall i \in N$. This proves the uniqueness of the solution to 225 Eq. (10).

226 To solve Eq.(10), we put forward a simple convergent iterative algorithm as 227 follows.

\section{Algorithm 1.}

Let $C_{k}=\left(c_{i j}{ }^{(k)}\right)_{n \times n}(k \in M)$ be the initial incomplete reciprocal preference relations provided by the DMs.

Step 1. Using Theorem 1 to judge whether an incomplete reciprocal preference relation $C_{k}(k \in M)$ given by the DM $e_{k}$ can be completed. If not, it is returned to expert $e_{k}$ for an updated reciprocal preference relation, otherwise, go to Step 2.

Step 2. Initiating the iteration by giving an initial priority vector $W(0)=$ $\left(w_{1}(0), w_{2}(0), \ldots, w_{n}(0)\right)^{T}$ and specifying an error parameter $\varepsilon \quad(0<\varepsilon<1)$, for example, $\varepsilon=0.0001$, and setting $L=0$.

\section{Step 3. Calculating}

$$
\eta_{i}(W(L))=\sum_{j=1}^{n} \sum_{k=1}^{m} h_{k} \delta_{j i}^{(k)} c_{j i}^{2(k)} \frac{w_{i}}{w_{j}}-\sum_{j=1}^{n} \sum_{k=1}^{m} h_{k} \delta_{i j}^{(k)} c_{i j}^{2(k)} \frac{w_{j}}{w_{i}}, \quad i \in N
$$

If $\left|\eta_{i}(W(L))\right| \leq \varepsilon$ holds for all $i \in N$, then $W^{*}=W(L)$ and stop, otherwise, continue to Step 4.

Step 4. Determining $p$ such that $\left|\eta_{p}(W(L))\right|=\max _{i \in N}\left\{\left|\eta_{i}(W(L))\right|\right\}$ and computing 


$$
T(L)=\sqrt{\frac{\sum_{j=1, j \neq p}^{n} \sum_{k=1}^{m} h_{k} \delta_{p j}^{(k)} c_{p j}^{2(k)} \frac{w_{j}(L)}{w_{p}(L)}}{\sum_{j=1, j \neq p}^{n} \sum_{k=1}^{m} h_{k} \delta_{j p}^{(k)} c_{j p}^{2(k)} \frac{w_{p}(L)}{w_{j}(L)}}}
$$

$$
f_{i}(L)= \begin{cases}T(L) w_{p}(L), & i=p, \\ w_{i}(L), & i \neq p\end{cases}
$$

$$
w_{i}(L+1)=f_{i}(L) / \sum_{\mathrm{i}=1}^{n} f_{i}(L), \quad i \in N \text {. }
$$

Step 5. Let $L=L+1$ and go to Step 3 .

For Algorithm 1, we can establish the following theorem.

Theorem 3. Algorithm 1 is convergent for any $\varepsilon>0$.

Proof. We shall examine how $F(W)$ changes, when $W(L)$ progresses to $W(L+1)$. Suppose that $t>0$ and $S(t)=F(f(L))=F\left(w_{1}(L), \ldots, w_{p-1}(L), t w_{p}(L), w_{p+1}(L), \ldots\right.$, $\left.w_{n}(L)\right)$.

Then we have

$$
\begin{aligned}
S(t)= & \sum_{j=1, j \neq p}^{n} \sum_{k=1}^{m} h_{k} \delta_{p j}^{(k)}\left[\left(c_{p j}^{(k)}-\frac{t w_{p}(L)}{t w_{p}(L)+w_{j}(L)}\right)^{2}\right] \frac{t w_{p}(L)+w_{j}(L)}{t w_{p}(L)} \\
& +\sum_{i=1, i \neq p}^{n} \sum_{k=1}^{m} h_{k} \delta_{i p}^{(k)}\left[\left(c_{i p}^{(k)}-\frac{w_{i}(L)}{w_{i}(L)+t w_{p}(L)}\right)^{2}\right] \frac{w_{i}(L)+t w_{p}(L)}{w_{i}(L)} \\
& +\sum_{i=1, i \neq p}^{n} \sum_{j=1, j \neq p}^{n} \sum_{k=1}^{m} h_{k} \delta_{i j}^{(k)}\left[\left(c_{i j}^{(k)}-\frac{w_{i}(L)}{w_{i}(L)+w_{j}(L)}\right)^{2}\right] \frac{w_{i}(L)+w_{j}(L)}{w_{i}(L)}
\end{aligned}
$$

which is equivalent to

$$
\begin{aligned}
S(t)= & \sum_{j=1, j \neq p}^{n} \sum_{k=1}^{m} h_{k} \delta_{p j}^{(k)} c_{p j}^{2(k)} \frac{w_{j}(L)}{w_{p}(L)} \cdot \frac{1}{t}+\sum_{j=1, j \neq p}^{n} \sum_{k=1}^{m} h_{k} \delta_{j p}^{(k)} c_{j p}^{2(k)} \frac{w_{p}(L)}{w_{j}(L)} \cdot t \\
& +\sum_{j=1, j \neq p}^{n} \sum_{k=1}^{m} h_{k}\left[\delta_{j p}^{(k)} c_{j p}^{2(k)}+\delta_{p j}^{(k)} c_{p j}^{2(k)}-2\left(\delta_{j p}^{(k)} c_{j p}^{(k)}+\delta_{p j}^{(k)} c_{p j}^{(k)}\right)\right]
\end{aligned}
$$




$$
\begin{aligned}
& +\sum_{j=1, j \neq p}^{n} \sum_{k=1}^{m} h_{k}\left(\delta_{j p}^{(k)} \frac{t w_{p}(L)}{t w_{p}(L)+w_{j}(L)}+\delta_{p j}^{(k)} \frac{w_{j}(L)}{t w_{p}(L)+w_{j}(L)}\right) \\
& +\sum_{i=1, i \neq p}^{n} \sum_{j=1, j \neq p}^{n} \sum_{k=1}^{m} h_{k} \delta_{i j}^{(k)}\left[\left(c_{i j}^{(k)}-\frac{w_{i}(L)}{w_{i}(L)+w_{j}(L)}\right)^{2}\right] \frac{w_{i}(L)+w_{j}(L)}{w_{i}(L)}
\end{aligned}
$$

$$
\begin{aligned}
& q_{1}=\sum_{j=1, j \neq p}^{n} \sum_{k=1}^{m} h_{k} \delta_{p j}^{(k)} c_{p j}^{2(k)} \frac{w_{j}(L)}{w_{p}(L)}, \\
& q_{2}=\sum_{j=1, j \neq p}^{n} \sum_{k=1}^{m} h_{k} \delta_{j p}^{(k)} c_{j p}^{2(k)} \frac{w_{p}(L)}{w_{j}(L)},
\end{aligned}
$$$$
q_{3}=\sum_{j=1, j \neq p}^{n} \sum_{k=1}^{m} h_{k}\left[\delta_{j p}^{(k)} c_{j p}^{2(k)}+\delta_{p j}^{(k)} c_{p j}^{2(k)}-2\left(\delta_{j p}^{(k)} c_{j p}^{(k)}+\delta_{p j}^{(k)} c_{p j}^{(k)}\right)\right]
$$$$
+\sum_{j=1, j \neq p}^{n} \sum_{k=1}^{m} h_{k}\left(\delta_{j p}^{(k)} \frac{t w_{p}(L)}{t w_{p}(L)+w_{j}(L)}+\delta_{p j}^{(k)} \frac{w_{j}(L)}{t w_{p}(L)+w_{j}(L)}\right)
$$$$
+\sum_{i=1, i \neq p}^{n} \sum_{j=1, j \neq p}^{n} \sum_{k=1}^{m} h_{k} \delta_{i j}^{(k)}\left[\left(c_{i j}^{(k)}-\frac{w_{i}(L)}{w_{i}(L)+w_{j}(L)}\right)^{2}\right] \frac{w_{i}(L)+w_{j}(L)}{w_{i}(L)}
$$

Since $\delta_{i j}^{(k)}=\delta_{j i}^{(k)}$, the second double summation term in Eq. (29) can be rewritten as

$$
\begin{aligned}
& \sum_{j=1, j \neq p}^{n} \sum_{k=1}^{m} h_{k}\left(\delta_{j p}^{(k)} \frac{t w_{p}(L)}{t w_{p}(L)+w_{j}(L)}+\delta_{p j}^{(k)} \frac{w_{j}(L)}{t w_{p}(L)+w_{j}(L)}\right) \\
& = \begin{cases}(n-1) & \text { if } \delta_{j p}^{(k)}=\delta_{p j}^{(k)}=1 \\
0 & \text { if } \delta_{j p}^{(k)}=\delta_{p j}^{(k)}=0\end{cases}
\end{aligned}
$$

Therefore, $q_{3}$ can be further simplified as

$$
\begin{aligned}
q_{3}= & \sum_{j=1, j \neq p}^{n} \sum_{k=1}^{m} h_{k}\left[\delta_{j p}^{(k)} c_{j p}^{2(k)}+\delta_{p j}^{(k)} c_{p j}^{2(k)}-2\left(\delta_{j p}^{(k)} c_{j p}^{(k)}+\delta_{p j}^{(k)} c_{p j}^{(k)}\right)\right] \\
& +\sum_{i=1, i \neq p}^{n} \sum_{j=1, j \neq p}^{n} \sum_{k=1}^{m} h_{k} \delta_{i j}^{(k)}\left[\left(c_{i j}^{(k)}-\frac{w_{i}(L)}{w_{i}(L)+w_{j}(L)}\right)^{2}\right] \frac{w_{i}(L)+w_{j}(L)}{w_{i}(L)}
\end{aligned}
$$




$$
+ \begin{cases}(n-1) & \text { if } \delta_{j p}^{(k)}=\delta_{p j}^{(k)}=1 \\ 0 & \text { if } \delta_{j p}^{(k)}=\delta_{p j}^{(k)}=0\end{cases}
$$

275

276

277

278

280

281

282

283

284

286

287

288

289

290

291

292

This indicates that $q_{3}$ is independent of $t$. Then Eq. (26) can be equivalently expressed as

$$
S(t)=q_{1} / t+q_{2} \cdot t+q_{3} .
$$

By setting $\frac{d S(t)}{d t}$ to be zero, we have

$$
t^{*}=\sqrt{q_{1} / q_{2}}=\sqrt{\frac{\sum_{j=1, j \neq p}^{n} \sum_{k=1}^{m} h_{k} \delta_{p j}^{(k)} c_{p j}^{2(k)} \frac{w_{j}(L)}{w_{p}(L)}}{\sum_{j=1, j \neq p}^{n} \sum_{k=1}^{m} h_{k} \delta_{j p}^{(k)} c_{j p}^{2(k)} \frac{w_{p}(L)}{w_{j}(L)}}},
$$

where $t^{*}$ stands for the minimum point, and $S\left(t^{*}\right)$ gives the minimum value of $S(t)$

If $t^{*}=1$, Eq. (33) is equivalent to

$$
\sum_{j=1, j \neq p}^{n} \sum_{k=1}^{m} h_{k} \delta_{p j}^{(k)} c_{p j}^{2(k)} \frac{w_{j}(L)}{w_{p}(L)}=\sum_{j=1, j \neq p}^{n} \sum_{k=1}^{m} h_{k} \delta_{j p}^{(k)} c_{j p}^{2(k)} \frac{w_{p}(L)}{w_{j}(L)} .
$$

which also holds for $j=p$, therefore, we have

$$
\sum_{j=1}^{n} \sum_{k=1}^{m} h_{k} \delta_{p j}^{(k)} c_{p j}^{2(k)} \frac{w_{j}(L)}{w_{p}(L)}=\sum_{j=1}^{n} \sum_{k=1}^{m} h_{k} \delta_{j p}^{(k)} c_{j p}^{2(k)} \frac{w_{p}(L)}{w_{j}(L)} .
$$

That is

$$
\eta_{p}(W(L))=\sum_{j=1}^{n} \sum_{k=1}^{m} h_{k} \delta_{j p}^{(k)} c_{j p}^{2(k)} \frac{w_{p}(L)}{w_{j}(L)}-\sum_{j=1}^{n} \sum_{k=1}^{m} h_{k} \delta_{p j}^{(k)} c_{p j}^{2(k)} \frac{w_{j}(L)}{w_{p}(L)}=0 .
$$

By the definition of $p$ in Step 3, we have $\left|\eta_{p}(W(L))\right|=0$. Since $p$ is the subscript such that $\left|\eta_{i}(W(L))\right|$ is maximized, we thus have $\left|\eta_{i}(W(L))\right|=0$ for all $i \in N$.

Therefore, the algorithm terminates and $W^{*}=W(L)$.

If $t^{*} \neq 1$, then 


$$
F(W(L))-F(f(L))=S(1)-S\left(t^{*}\right)=q_{1}+q_{2}-2 \sqrt{q_{1} q_{2}}=\left(\sqrt{q_{1}}-\sqrt{q_{2}}\right)^{2}>0
$$

Since $F(W)$ is a homogenous function, $F(f(L))=F(W(L+1))$. Inequality (37)

shows that $F(W(L+1))<F(W(L))$, for any $L \geq 0$. Therefore, $F(W(L))$ is a monotonically decreasing sequence with an infimum in $D_{w}$ and, hence, convergent.

\section{A method for repairing inconsistency of incomplete reciprocal preference relations}

If the consistency level of an incomplete reciprocal preference relation is too low and deemed unacceptable, it can be returned to the DM for a reassessment until the updated one reaches an acceptable consistency level. This approach is presumably more reliable and accurate, but it is often impracticable because the iteration process can be tedious and time-consuming. To facilitate the decision process, this section puts forwards an automated procedure to improve the consistency level of a given incomplete reciprocal preference relation with unacceptable consistency $F C R \geq 0.1$. Whenever possible, the DM's intervention should be called upon, but this procedure serves as a convenient tool and can be employed by the analyst to facilitate the DM in eliciting his/her preference expeditiously. We first introduce the consistency deviation variable for $c_{i j}$ as follows.

$$
d_{i j}=\left|c_{i j}-w_{i} /\left(w_{i}+w_{j}\right)\right|
$$

If $d_{i j} \equiv 0$, for all $i, j \in N$ and $c_{i j} \neq-$, then $C$ is a perfectly consistent incomplete reciprocal preference relation. The priority vector $W$ is able to precisely represent $C$. The higher the deviation $d_{i j}$, the more likely $c_{i j}$ should be updated.

Conceptually, a judgment $c_{i j}$ should be as close to $w_{i} /\left(w_{i}+w_{j}\right)$ as possible to make it more consistent. In addition, the known element $c_{i j}$ given by the expert often falls in the set $U=\{0,0.1,0.2,0.3,0.4,0.5,0.6,0.7,0.8,0.9,1\}$. To avoid 
excessive distortion of the DM's original judgment, the improved preference relation should not only increase the consistency level but also try to preserve the initial preference information. The procedure starts with identifying the unusual and false elements (UFEs) that are the most inconsistent element with the biggest $d_{i j}$. Once the UFEs are identified, the initial UFE $\left(c_{i j}\right)$ will be updated with $c_{i j}^{\prime}$, where $c_{i j}^{\prime}=\operatorname{round}\left(w_{i} /\left(w_{i}+w_{j}\right) \times 10\right) \times 10^{-1}$ and "round" is the usual round operation. This function ensures the updated judgment values are between 0 and 1 and have one decimal place. It is trivial to make adjustment to accommodate the case when the analyst or DM prefers to express the judgment in more decimal places.

Given the aforesaid discussion, the following algorithm is devised to repair inconsistency of an incomplete reciprocal preference relation.

\section{Algorithm 2}

Let $C=\left(c_{i j}\right)_{n \times n}$ be an incomplete reciprocal preference relation given by the DM.

Step 1. Using the CSM algorithm in Section 3 to obtain the priority vector $W=\left(w_{1}, w_{2}, \ldots w_{n}\right)^{T}$.

Step 2. Determining the consistency ratio of the incomplete reciprocal preference relation as per Eq. (1), if $F C R<0.1$, go to Step 5, otherwise, go to Step 3.

Step 3. Computing deviations $d_{i j}$ 's by using Eq. (38), and identifying the maximum deviation to find the corresponding UFEs.

Step 4. Updating the UFEs $\left(c_{i j}\right)$ with $c_{i j}^{\prime}$, where $c_{i j}^{\prime}=\operatorname{round}\left(w_{i} /\left(w_{i}+w_{j}\right) \times 10\right)$ $\times 10^{-1}$, and go to Step 1 .

Step 5. Ranking the alternatives according the priority vector $W^{*}$.

Step 6. End.

\section{Illustrative examples}

In this section, four numerical examples are examined to demonstrate the applications and advantages of the proposed CSM framework. Example 1 is a GDM 
problem with incomplete reciprocal preference relations and a comparative analysis is conducted between CSM and three existing methods. Example 2 is a single incomplete reciprocal preference relation with unacceptable consistency and Algorithm 2 is utilized to repair it until its consistency becomes acceptable. Example 3 considers a single incomplete reciprocal preference relation with acceptable consistency. The purpose is to compare the result derived from CSM with those from EM, NRAM, GPM, LSM and LLSM on three performance evaluation criteria: $F C R$, MAD and MD. Example 4 discusses a GDM problem with incomplete reciprocal preference relations with a purpose to show the advantages of CSM.

Example 1. For a GDM problem with four decision alternatives $x_{i}(i=1,2,3,4)$ and three DMs $e_{k}(k=1,2,3)$. The DMs provide their preferences over the four decision alternatives as three incomplete reciprocal preference relations [41].

$C_{1}=\left[\begin{array}{cccc}0.5 & 0.6 & - & 0.7 \\ 0.4 & 0.5 & 0.2 & 0.8 \\ - & 0.8 & 0.5 & 0.4 \\ 0.3 & 0.2 & 0.6 & 0.5\end{array}\right], C_{2}=\left[\begin{array}{cccc}0.5 & 0.8 & 0.4 & - \\ 0.2 & 0.5 & 0.3 & 0.6 \\ 0.6 & 0.7 & 0.5 & 0.3 \\ - & 0.4 & 0.7 & 0.5\end{array}\right], C_{3}=\left[\begin{array}{cccc}0.5 & 0.3 & 0.4 & 0.6 \\ 0.7 & 0.5 & - & 0.5 \\ 0.6 & - & 0.5 & 0.7 \\ 0.4 & 0.5 & 0.3 & 0.5\end{array}\right]$. $\mathrm{Xu}$ [41] employed goal programming (GP) models to derive a priority vector $W^{*}=(0.265,0.236,0.276,0.223)^{T}$ from the aforesaid three incomplete reciprocal preference relations. The research leads to a final ranking: $x_{3} \succ x_{1} \succ x_{2} \succ x_{4}$, which is the same as the ranking generated by the logarithmic least square method (LLSM) [38] but slightly differs from the one obtained by the least-square method (LSM) [17] with the order between $x_{2}$ and $x_{4}$ being reversed. We now examine the problem using the CSM. In order to offer a fair comparison with Xu [41]'s method, we also set $h_{1}=$ $h_{2}=h_{3}=1 / 3$.

Step 1. According to Theorem 1, we know that $C_{k}(k=1,2,3)$ can all be completed by known elements.

Step 2. Given an initial priority vector $W(0)=(0.25,0.25,0.25,0.25)^{T}$, specify the 
parameter $\varepsilon=0.1$, and let $L=0$.

Step 3. Calculate $\eta_{i}(W(0))$, we have

$$
\begin{aligned}
& \left|\eta_{1}(W(0))\right|=0.2>\varepsilon,\left|\eta_{2}(W(0))\right|=0.2>\varepsilon, \\
& \left|\eta_{3}(W(0))\right|=0.4>\varepsilon,\left|\eta_{4}(W(0))\right|=0.4>\varepsilon .
\end{aligned}
$$

As $\left|\eta_{i}(W(0))\right|>\varepsilon$ holds for all $i=1,2,3,4$, we continue to Step 4 .

Step 4. Determine $p$ such that $\left|\eta_{p}(W(L))\right|=\max _{i \in N}\left\{\left|\eta_{i}(W(L))\right|\right\}$, we can set $p=3$, and compute $T(0), f(0)$ and $W(1)$.

$$
T(0)=1.3650, \quad f(0)=(0.2500,0.2500,0.3413,0.2500)^{T},
$$$$
W(1)=(0.2291,0.2291,0.3127,0.2291)^{T} .
$$

Step 5. Let $L=L+1=1$ and go to Step 3 .

The computation processes are detailed in Table 2. It is clear that iterations terminates at $L=3$, when $\left|\eta_{1}\right|=0.0296<0.1,\left|\eta_{2}\right|=0.0072<0.1,\left|\eta_{3}\right|=0.0227<0.1$, $\left|\eta_{4}\right|=2.6357 \times 10^{-4}<0.1$, indicating that the derived priority vector has reached an acceptable level of $\varepsilon$. The optimal priority vector is thus found to be $W^{*}=$ $(0.2797,0.2197,0.3,0.2007)^{T}$, resulting in a ranking of the four alternatives $x_{3} \succ x_{1} \succ x_{2} \succ x_{4}$.

Remark 1. Computation results in Table 2 demonstrate that $F(W(L))$ decreases in iteration step $L$. However, for $\left|\eta_{i}(W(L))\right|$, this monotonicity does not hold any more and there may have ups and downs when $L$ increases, but eventually $\left|\eta_{i}(W(L))\right|$ will decrease to a value below the threshold $\varepsilon$ as ascertained by Theorem 3. As three of the four aforesaid methods derive an identical ranking with the other one yielding a slightly different order, this result demonstrates the robustness and credibility of the proposed CSM framework. To further compare the performance with the other three methods in fitting the three incomplete reciprocal preference relations, the following evaluation criteria are introduced: 
Maximum deviation (MD) for incomplete reciprocal preference relations

$$
\mathrm{MD}=\max _{i, j, k}\left\{\delta_{i j}^{(k)}\left(\frac{c_{i j}}{c_{j i}} \frac{w_{j}}{w_{i}}+\frac{c_{j i}}{c_{i j}} \frac{w_{i}}{w_{j}}-2\right) \mid i, j \in N, k \in M\right\}
$$

Maximum absolute deviation (MAD) for incomplete reciprocal preference relations

$$
\mathrm{MAD}=\max _{i, j, k}\left\{\delta_{i j}^{(k)} \mid c_{i j}^{(k)}-\frac{w_{i}}{w_{i}+w_{j}} \| i, j \in N, k \in M\right\} .
$$

where $\delta_{i j}^{(k)}$ is defined by Eq. (6). $d_{i j}^{(k)}=c_{i j}^{(k)}-w_{i} /\left(w_{i}+w_{j}\right)$ is the consistency

deviation for $c_{i j}^{(k)}$ in the incomplete reciprocal preference relation $C^{(k)}=\left(c_{i j}^{(k)}\right)_{n \times n}$. If the priority vector $W=\left(w_{1}, \ldots w_{n}\right)^{T}$ is able to precisely characterize the reciprocal preference relation $C^{(k)}$, then $\left|d_{i j}^{(k)}\right| \equiv 0$, otherwise, $\left|d_{i j}^{(k)}\right|>0$.

Table 3 indicates that CSM results in an identical ranking as GPM and LLSM while the ranking derived by LSM is slightly different. CSM has a comparable MAD as GPM, which is smaller than both LSM and LLSM. In terms of MD, CSM outperforms all the other three methods as it yields the smallest deviation. This partly shows the advantage of the CSM.

Remark 2. To facilitate a comparative study with GPM, LSM and LLSM, the weights of three reciprocal preference relations were set to be equal $\left(h_{1}=h_{2}=h_{3}=1 / 3\right)$. However, CSM allows an analyst to set different weights as per the practical situation, to properly reflect different experts' varying influences in the GDM problem at hand. It is worth noting that if $\delta_{i j}=1$, for all $i, j \in N$, then the proposed CSM can still be utilized to derive a priority vector from reciprocal preference relations. This means that CSM can be used for both complete and incomplete reciprocal preference relations. In addition, by setting $h_{1}=1$ and $h_{k}=0$, for $k=2, \ldots, m$, the CSM can be conveniently applied to derive a priority vector from a single incomplete reciprocal preference relation. This allows CSM to be used for a single expert's decision making problems in Examples 2 and 3. 
417 Furthermore, by using Algorithm 1, we can get the values of $L, W, F(W)$ and the 418 ranking of alternatives for different $\varepsilon$ 's as listed in Table 4. 
Table 2

The iterative processes for Example 1.

\begin{tabular}{|c|c|c|c|c|c|c|c|c|c|}
\hline Iterative steps & & & $L))$ & & & & & & $F(W)$ \\
\hline$L$ & $\left|\eta_{1}\right|$ & $\left|\eta_{2}\right|$ & $\left|\eta_{3}\right|$ & $\left|\eta_{4}\right|$ & $w_{1}$ & $w_{2}$ & $w_{3}$ & $w_{4}$ & \\
\hline 0 & 0.2 & 0.2 & 0.4 & 0.4 & 0.25 & 0.25 & 0.25 & 0.25 & 0.7067 \\
\hline 1 & 0.3031 & 0.0835 & $1.156 \times 10^{-4}$ & 0.2197 & 0.2291 & 0.2291 & 0.3127 & 0.2291 & 0.6449 \\
\hline 2 & $6.197 \times 10^{-5}$ & 0.0571 & 0.0792 & 0.1362 & 0.2744 & 0.2256 & 0.2943 & 0.2156 & 0.6086 \\
\hline 3 & 0.0296 & 0.0072 & 0.0227 & $2.6357 \times 10^{-4}$ & 0.2797 & 0.2197 & 0.3000 & 0.2007 & 0.6024 \\
\hline
\end{tabular}

Table 3

Performance comparisons for Example 1.

\begin{tabular}{|c|c|c|c|c|}
\hline Methods & $W^{*}$ & Ranking & MD & MAD \\
\hline CSM (this article) & $(0.2797,0.2197,0.3000,0.2007)^{T}$ & $x_{3} \succ x_{1} \succ x_{2} \succ x_{4}$ & 1.9277 & 0.2992 \\
\hline LSM [17] & $(0.2822,0.1968,0.3202,0.2009)^{T}$ & $x_{3} \succ x_{1} \succ x_{4} \succ x_{2}$ & 2.3282 & 0.3145 \\
\hline GPM [41] & $(0.265,0.236,0.276,0.223)^{T}$ & $x_{3} \succ x_{1} \succ x_{2} \succ x_{4}$ & 2.0442 & 0.2858 \\
\hline LLSM [38] & $(0.2806,0.2105,0.3189,0.1900)^{T}$ & $x_{3} \succ x_{1} \succ x_{2} \succ x_{4}$ & 2.1717 & 0.3266 \\
\hline
\end{tabular}

\section{Table 4}

The values of $L, W, F(W)$ and ranking order for different $\varepsilon$ in Example 1.

\begin{tabular}{|c|c|c|c|c|c|c|c|c|}
\hline$\varepsilon$ & $L$ & $W$ & Ranking & $F(W)$ & $\left|\eta_{1}\right|$ & $\left|\eta_{2}\right|$ & $\left|\eta_{3}\right|$ & $\left|\eta_{4}\right|$ \\
\hline $10^{-1}$ & 3 & $(0.2797,0.2197,0.3000,0.2007)^{T}$ & $x_{3} \succ x_{1} \succ x_{2} \succ x_{4}$ & 0.6024 & 0.0001 & 0.0728 & 0.0868 & 0.0141 \\
\hline $10^{-2}$ & 15 & $(0.2753,0.2204,0.3032,0.2011)^{T}$ & $x_{3} \succ x_{1} \succ x_{2} \succ x_{4}$ & 0.6020 & 0.0031 & 0 & 0.0083 & 0.0052 \\
\hline $10^{-3}$ & 24 & $(0.2746,0.2201,0.3041,0.2012)^{T}$ & $x_{3} \succ x_{1} \succ x_{2} \succ x_{4}$ & 0.6019 & $3.6858 \times 10^{-4}$ & 0 & $9.8063 \times 10^{-4}$ & $6.1205 \times 10^{-4}$ \\
\hline $10^{-4}$ & 34 & $(0.2745,0.2201,0.3043,0.2012)^{T}$ & $x_{3} \succ x_{1} \succ x_{2} \succ x_{4}$ & 0.6019 & $5.9215 \times 10^{-5}$ & $2.6310 \times 10^{-5}$ & $8.5525 \times 10^{-5}$ & 0 \\
\hline $10^{-5}$ & 44 & $(0.2746,0.2201,0.3041,0.2012)^{T}$ & $x_{3} \succ x_{1} \succ x_{2} \succ x_{4}$ & 0.6019 & $1.1102 \times 10^{-16}$ & $6.4088 \times 10^{-6}$ & $8.2368 \times 10^{-6}$ & $1.8281 \times 10^{-6}$ \\
\hline $10^{-6}$ & 53 & $(0.2746,0.2201,0.3041,0.2012)^{T}$ & $x_{3} \succ x_{1} \succ x_{2} \succ x_{4}$ & 0.6019 & $1.1102 \times 10^{-16}$ & $6.4088 \times 10^{-6}$ & $8.2368 \times 10^{-6}$ & $1.8281 \times 10^{-6}$ \\
\hline
\end{tabular}


404

Example 2. Consider a single DM's decision problem with six alternatives $x_{i}$ $(i=1,2, \ldots, 6)$. The DM provides his/her preferences over the six decision alternatives, as an incomplete reciprocal preference relation which is shown below (adapted from [42]).

$$
C=\left[\begin{array}{cccccc}
0.5 & - & - & 0.3 & 0.8 & 0.3 \\
- & 0.5 & - & - & - & - \\
- & - & 0.5 & - & - & - \\
0.7 & - & - & 0.5 & 0.4 & 0.8 \\
0.2 & - & - & 0.6 & 0.5 & 0.7 \\
0.7 & - & - & 0.2 & 0.3 & 0.5
\end{array}\right]
$$

Step 1. According to Theorem 1, it is easy to tell that $C$ can be completed as no non-diagonal elements are furnished in the second or third row (column) of $C$. Therefore, the initial judgment matrix has to be returned to the DM for an update, resulting in the following incomplete reciprocal preference relation:

$$
C=\left[\begin{array}{cccccc}
0.5 & 0.3 & - & 0.3 & 0.8 & 0.3 \\
0.7 & 0.5 & 0.7 & - & 0.6 & - \\
- & 0.3 & 0.5 & 0.4 & - & - \\
0.7 & - & 0.6 & 0.5 & 0.4 & 0.8 \\
0.2 & 0.4 & - & 0.6 & 0.5 & 0.7 \\
0.7 & - & - & 0.2 & 0.3 & 0.5
\end{array}\right]
$$

Without loss of generality, let the original weight vector be $W(0)=(1 / 6,1 / 6,1 / 6$, $1 / 6,1 / 6,1 / 6)^{T}$. Using Algorithm 1, one can get the values of $L, W, F(W), F C R$, $\left|\eta_{i}(W(L))\right|$ and ranking results by setting different $\varepsilon$ values as listed in Table 5. When $\varepsilon$ is sufficiently small, the weight vector approaches

$$
W^{*}=(0.1301,0.2714,0.1281,0.2090,0.1509,0.1106)^{T},
$$

Step 2. Computing FCR by Eq. (1).

$$
F C I=0.1870, F C R=F C I / R I=0.1870 / 1.26=0.1484>0.1 .
$$

Since $F C R>0.1$, the incomplete reciprocal preference relation $C$ does not possess satisfactory consistency. We need to find its UFEs to repair this preference relation. 

corresponding consistent representation, we have

$$
D=\left[\begin{array}{cccccc}
0 & 0.0241 & 0 & 0.0837 & 0.3369 & 0.2405 \\
0.0241 & 0 & 0.0206 & 0 & 0.0427 & 0 \\
0 & 0.0206 & 0 & 0.0201 & 0 & 0 \\
0.0837 & 0 & 0.0201 & 0 & 0.1808 & 0.1461 \\
0.3369 & 0.0427 & 0 & 0.1808 & 0 & 0.1231 \\
0.2405 & 0 & 0 & 0.1461 & 0.1231 & 0
\end{array}\right] .
$$

Obviously, the maximum deviations are $d_{15}$ and $d_{51}$, so the UFEs are $c_{15}$ and $c_{51}$.

Thus $C$ is updated as

$$
C^{\prime}=\left[\begin{array}{cccccc}
0.5 & 0.3 & - & 0.3 & 0.5 & 0.3 \\
0.7 & 0.5 & 0.7 & - & 0.6 & - \\
- & 0.3 & 0.5 & 0.4 & - & - \\
0.7 & - & 0.6 & 0.5 & 0.4 & 0.8 \\
0.5 & 0.4 & - & 0.6 & 0.5 & 0.7 \\
0.7 & - & - & 0.2 & 0.3 & 0.5
\end{array}\right]
$$

Using Algorithm 1, one can obtain the values of $L, W, F(W), F C R,\left|\eta_{i}(W(L))\right|$ and ranking of alternatives with different $\varepsilon$ 's as listed in Table 6 . When $\varepsilon$ is sufficiently small, the final priority vector is obtained as

$$
W^{*}=(0.0994,0.2699,0.1275,0.2083,0.1889,0.1061)^{T}
$$

Computing FCR by Eq. (1).

$$
F C I=0.0661, \quad F C R=F C I / R I=0.0661 / 1.26=0.0525<0.1 .
$$

Thus, this updated $C$ is deemed to have acceptable consistency.

Step 5. Using the final priority vector $W^{*}$ to rank the alternatives as

$$
W^{*}=(0.0994,0.2699,0.1275,0.2083,0.1889,0.1061)^{T} .
$$

$$
x_{2} \succ x_{4} \succ x_{5} \succ x_{3} \succ x_{6} \succ x_{1} .
$$


441 By changing only $c_{15}$ and $c_{51}$, we were able to rectify an incomplete reciprocal 442 preference relation to derive one with acceptable consistency. This allows the analyst 443 to avoid the hassle of returning the inconsistent preference relation to the DM for 444 reconsideration.

445 Remark 3. Numerical results in Tables 4, 5 and 6 demonstrate that iteration step $L$ 446 increases when error parameter $\varepsilon$ decreases. In general, $F(W)$ and the consistency 447 ratio of an incomplete reciprocal preference relation $C$ gets smaller when $\varepsilon$ decrease. 448 When the error parameter $\varepsilon$ is sufficiently small, $W, F(W), F C R$, and ranking results 449 will converge to a set of values and remain unchanged.

450 In order to show the effectiveness of CSM, the other three methods EM [40], 451 LSM [17], and LLSM [38] are also applied to the rectified C' and assessed in terms of 452 the criteria FCR, MD and MAD. Table 7 lists the ranking results by the four methods. 453 It is clear that CSM and LLSM yield the same ranking $x_{2} \succ x_{4} \succ x_{5} \succ x_{3} \succ x_{6} \succ x_{1}$, 454 but EM and LSM generate slightly different rankings. Most notably, the EM and LSM 455 reverse the order of $x_{1}$ and $x_{6}$, while the DM's original judgment points to $x_{6} \succ x_{1}$ 456 because $c_{61}=c_{61}^{\prime}=0.7$. It is apparent that this reverse is unwarranted and undesirable. 457 Moreover, CSM produces the smallest MD and MAD among the four methods, and 458 the FCR from CSM is marginally larger than that from LLSM, but is smaller than 459 those derived from EM and LSM. Across the three metrics, FCR, MD and MAD, 460 Table 7 shows that CSM overall performs better than the other three methods EM, $461 \quad$ LSM and LLSM. 
Table 5. The values of $L, W, F(W)$, FCR and rankings for different $\varepsilon$ of $C$ in Example 2.

\begin{tabular}{|c|c|c|c|c|c|c|}
\hline$\varepsilon$ & $L$ & $W$ & Ranking & $F(W)$ & $F C R$ & $\left|\eta_{i}(W(L))\right|$ \\
\hline $10^{-1}$ & 26 & $(0.1319,0.2565,0.1302,0.2147,0.1540,0.1128)^{T}$ & $x_{2} \succ x_{4} \succ x_{5} \succ x_{1} \succ x_{3} \succ x_{6}$ & 1.0276 & 0.1490 & $4.4409 \times 10^{-16}, 0.098,0.0256,0.0093,0.0291,0.034$ \\
\hline $10^{-2}$ & 66 & $(0.1305,0.2699,0.1280,0.2094,0.1511,0.1110)^{T}$ & $x_{2} \succ x_{4} \succ x_{5} \succ x_{1} \succ x_{3} \succ x_{6}$ & 1.0239 & 0.1484 & $\begin{array}{c}0.0041,0.0095,1.1102 \times 10^{-16}, 0.0045,2.2204 \times 10^{-16}, \\
9.3303 \times 10^{-4}\end{array}$ \\
\hline $10^{-3}$ & 107 & $(0.1301,0.2712,0.1281,0.2090,0.1509,0.1107)^{T}$ & $x_{2} \succ x_{4} \succ x_{5} \succ x_{1} \succ x_{3} \succ x_{6}$ & 1.0239 & 0.1484 & $\begin{array}{l}4.2799 \times 10^{-4}, 9.7803 \times 10^{-4}, 2.2542 \times 10^{-4}, \\
2.2980 \times 10^{-4}, 2.2204 \times 10^{-16}, 9.4821 \times 10^{-5}\end{array}$ \\
\hline $10^{-4}$ & 146 & $(0.1301,0.2714,0.1281,0.2090,0.1509,0.1106)^{T}$ & $x_{2} \succ x_{4} \succ x_{5} \succ x_{1} \succ x_{3} \succ x_{6}$ & 1.0239 & 0.1484 & $\begin{array}{c}2.2204 \times 10^{-16}, 9.6242 \times 10^{-5}, 2.2204 \times 10^{-16} \\
3.3698 \times 10^{-5}, 2.8917 \times 10^{-5}, 3.3627 \times 10^{-5}\end{array}$ \\
\hline $10^{-5}$ & 187 & $(0.1301,0.2714,0.1281,0.2090,0.1509,0.1106)^{T}$ & $x_{2} \succ x_{4} \succ x_{5} \succ x_{1} \succ x_{3} \succ x_{6}$ & 1.0239 & 0.1484 & $\begin{array}{c}2.2204 \times 10^{-16}, 9.6242 \times 10^{-5}, 2.2204 \times 10^{-16}, \\
3.3698 \times 10^{-5}, 2.8917 \times 10^{-5}, 3.3627 \times 10^{-5}\end{array}$ \\
\hline $10^{-6}$ & 227 & $(0.1301,0.2714,0.1281,0.2090,0.1509,0.1106)^{T}$ & $x_{2} \succ x_{4} \succ x_{5} \succ x_{1} \succ x_{3} \succ x_{6}$ & 1.0239 & 0.1484 & $\begin{array}{c}2.2204 \times 10^{-16}, 9.6242 \times 10^{-5}, 2.2204 \times 10^{-16}, \\
3.3698 \times 10^{-5}, 2.8917 \times 10^{-5}, 3.3627 \times 10^{-5}\end{array}$ \\
\hline
\end{tabular}


Table 6. The values of $L, W, F(W)$, FCR and rankings for different $\varepsilon$ of $C^{\prime}$ in Example 2.

\begin{tabular}{|c|c|c|c|c|c|c|}
\hline$\varepsilon$ & $L$ & $W$ & Ranking & $F(W)$ & $F C R$ & $\left|\eta_{i}(W(L))\right|$ \\
\hline $10^{-1}$ & 22 & $(0.1014,0.2551,0.1288,0.2126,0.1914,0.1108)^{T}$ & $x_{2} \succ x_{4} \succ x_{5} \succ x_{3} \succ x_{6} \succ x_{1}$ & 0.4193 & 0.0532 & $0.0143,0.0943,0.0127,0.0406,0,0.0267$ \\
\hline $10^{-2}$ & 61 & $(0.0994,0.2685,0.1278,0.2086,0.1892,0.1064)^{T}$ & $x_{2} \succ x_{4} \succ x_{5} \succ x_{3} \succ x_{6} \succ x_{1}$ & 0.4154 & 0.0525 & $\begin{array}{c}2.2204 \times 10^{-16}, 0.0092,0.003,0.003 \\
0.0022,0.001\end{array}$ \\
\hline $10^{-3}$ & 97 & $(0.0994,0.2698,0.1275,0.2083,0.1889,0.1061)^{T}$ & $x_{2} \succ x_{4} \succ x_{5} \succ x_{3} \succ x_{6} \succ x_{1}$ & 0.4154 & 0.0525 & $\begin{array}{c}2.2345 \times 10^{-4}, 8.7826 \times 10^{-4}, 0,2.2196 \times 10^{-4} \\
1.0941 \times 10^{-4}, 3.2344 \times 10^{-4}\end{array}$ \\
\hline $10^{-4}$ & 133 & $(0.0994,0.2699,0.1275,0.2083,0.1889,0.1061)^{T}$ & $x_{2} \succ x_{4} \succ x_{5} \succ x_{3} \succ x_{6} \succ x_{1}$ & 0.4154 & 0.0525 & $\begin{array}{l}2.1921 \times 10^{-5}, 9.2656 \times 10^{-5}, 1.1102 \times 10^{-16}, \\
3.8488 \times 10^{-5}, 2.2204 \times 10^{-16}, 3.2246 \times 10^{-5}\end{array}$ \\
\hline $10^{-5}$ & 170 & $(0.0994,0.2699,0.1275,0.2082,0.1889,0.1061)^{T}$ & $x_{2} \succ x_{4} \succ x_{5} \succ x_{3} \succ x_{6} \succ x_{1}$ & 0.4154 & 0.0525 & $\begin{array}{c}2.9494 \times 10^{-6}, 9.6250 \times 10^{-6}, 1.3882 \times 10^{-6} \\
1.5475 \times 10^{-6}, 0,3.7399 \times 10^{-6}\end{array}$ \\
\hline $10^{-6}$ & 209 & $(0.0994,0.2699,0.1275,0.2082,0.1889,0.1061)^{T}$ & $x_{2} \succ x_{4} \succ x_{5} \succ x_{3} \succ x_{6} \succ x_{1}$ & 0.4154 & 0.0525 & $\begin{array}{c}0,9.2517 \times 10^{-7}, 2.4979 \times 10^{-7}, 1.9570 \times 10^{-7} \\
3.6807 \times 10^{-7}, 1.1160 \times 10^{-7}\end{array}$ \\
\hline
\end{tabular}

Table 7. Performance comparisons for Example 2.

\begin{tabular}{|c|c|c|c|c|c|}
\hline Method & $W^{*}$ & Ranking & $F C R$ & MD & MAD \\
\hline CSM & $(0.0994,0.2699,0.1275,0.2083,0.1889,0.1061)^{T}$ & $x_{2} \succ x_{4} \succ x_{5} \succ x_{3} \succ x_{6} \succ x_{1}$ & 0.0525 & 0.6434 & 0.1837 \\
\hline $\mathrm{EM}$ & $(0.1038,0.2780,0.1262,0.2017,0.1949,0.0953)^{T}$ & $x_{2} \succ x_{4} \succ x_{5} \succ x_{3} \succ x_{1} \succ x_{6}$ & 0.054 & 0.9349 & 0.2213 \\
\hline LSM & $(0.1017,0.3036,0.1354,0.1849,0.1937,0.0808)^{T}$ & $x_{2} \succ x_{5} \succ x_{4} \succ x_{3} \succ x_{1} \succ x_{6}$ & 0.0607 & 1.2774 & 0.2573 \\
\hline LLSM & $(0.0965,0.2682,0.1288,0.2166,0.1901,0.0998)^{T}$ & $x_{2} \succ x_{4} \succ x_{5} \succ x_{3} \succ x_{6} \succ x_{1}$ & 0.0519 & 0.6994 & 0.1916 \\
\hline
\end{tabular}


Example 3. Given a decision problem with six alternatives $x_{i}(i=1,2, \ldots, 6)$, the DM provides his/her preferences over the six decision alternatives, as an incomplete reciprocal preference relation (adapted from [38])

$$
C=\left[\begin{array}{cccccc}
0.5 & 0.4 & - & 0.3 & 0.3 & 0.3 \\
0.6 & 0.5 & 0.6 & 0.5 & - & 0.4 \\
- & 0.4 & 0.5 & 0.3 & 0.6 & - \\
0.7 & 0.5 & 0.7 & 0.5 & 0.4 & 0.8 \\
0.7 & - & 0.4 & 0.6 & 0.5 & 0.7 \\
0.7 & 0.6 & - & 0.2 & 0.3 & 0.5
\end{array}\right]
$$

This incomplete reciprocal preference relation was investigated by $\mathrm{Xu}$ et al. [38], in which the optimal priority vector is derived by LLSM as $W^{*}=(0.0878,0.1599$, $0.1551,0.2464,0.2208,0.1301)^{T}$. This yields a ranking of the six alternatives $x_{4} \succ x_{5} \succ x_{2} \succ x_{3} \succ x_{6} \succ x_{1}$. We now examine the problem using CSM as follows.

According to Theorem 1, we know that $C$ can be completed. Without loss of generality, we set the original weight vector as $W(0)=(1 / 6,1 / 6,1 / 6,1 / 6,1 / 6,1 / 6)^{T}$.

When $\varepsilon$ is set to $10^{-3}$, the values of $W, F(W), F C R$ and ranking of alternatives will stabilize and remain unchanged. At $L=55$, one has $F(W)=0.5860,\left|\eta_{1}\right|=1.1863 \times 10^{-5}<$ $\varepsilon,\left|\eta_{2}\right|=0<\varepsilon,\left|\eta_{3}\right|=1.2503 \times 10^{-5}<\varepsilon,\left|\eta_{4}\right|=9.6445 \times 10^{-5}<\varepsilon,\left|\eta_{5}\right|=2.9292 \times 10^{-5}<\varepsilon$, $\left|\eta_{6}\right|=4.2788 \times 10^{-5}<\varepsilon \quad, \quad F C R=0.0728, \quad W^{*}=(0.0884,0.1615,0.1581,0.2365,0.2185$, $0.1370)^{T}$, implying a ranking of these six alternatives as: $x_{4} \succ x_{5} \succ x_{2} \succ x_{3} \succ x_{6} \succ x_{1}$. For this single incomplete reciprocal preference relation, it can also be solved by EM [40], NRAM[32], LSM[17], LLSM[38] and GPM [41]. The results are shown in Table 8, from which we can see that CSM achieves the same ranking as EM, NRAM, LSM and LLSM, $x_{4} \succ x_{5} \succ x_{2} \succ x_{3} \succ x_{6} \succ x_{1}$, while GPM yields a slightly different ranking, $x_{4} \sim x_{5} \succ x_{2} \succ x_{3} \sim x_{6} \sim x_{1}$, which fails to discriminate $x_{4}$ and $x_{5}$, as well as $x_{1}, x_{3}$ and $x_{6}$. Furthermore, both NRAM and GPM lead to unacceptable 
consistency ratio $F C R>0.1$, and have larger MD and MAD values than other methods. A further examination reveals that CSM results in the smallest MAD value among these six methods and outperforms NRAM, GPM and LSM in all the three criteria.

Example 4. Consider a GDM problem with three DMs providing the following incomplete reciprocal preference relations $C_{i} \quad(i=1,2,3)$ for a set of four alternatives $X=\left\{x_{1}, x_{2}, x_{3}, x_{4}\right\}:$

$C_{1}=\left[\begin{array}{cccc}0.5 & 0.3 & - & 0.5 \\ 0.7 & 0.5 & 0.6 & 0.6 \\ - & 0.4 & 0.5 & 0.7 \\ 0.5 & 0.4 & 0.3 & 0.5\end{array}\right], C_{2}=\left[\begin{array}{cccc}0.5 & 0.2 & 0.6 & 0.7 \\ 0.8 & 0.5 & 0.8 & - \\ 0.4 & 0.2 & 0.5 & 0.8 \\ 0.3 & - & 0.4 & 0.5\end{array}\right], C_{3}=\left[\begin{array}{cccc}0.5 & 0.2 & 0.5 & 0.6 \\ 0.8 & 0.5 & - & 0.7 \\ 0.5 & - & 0.5 & 0.8 \\ 0.4 & 0.3 & 0.2 & 0.5\end{array}\right]$. Let $h_{1}=h_{2}=h_{3}=1 / 3$ and $\varepsilon=0.0001$. After several iterations, $\left|\eta_{1}\right|=5.7495 \times$ $10^{-5}<\varepsilon,\left|\eta_{2}\right|=8.8394 \times 10^{-5}<\varepsilon,\left|\eta_{3}\right|=3.0899 \times 10^{-5}<\varepsilon, \quad\left|\eta_{4}\right|=1.1102 \times 10^{-16}<\varepsilon$, indicating that the derived priority vector has reached an acceptable error level. Therefore, the optimal priority vector is found to be $W^{*}=(0.1954,0.4386$, $0.2316,0.1344)^{T}$

The comparative result is shown in Table 9. It is clear that CSM preforms the best in both MD and MAD. CSM obtains the same ranking as LLSM and LSM, $x_{2} \succ x_{3} \succ x_{1} \succ x_{4}$, while GPM yields a slightly different ranking $x_{2} \succ x_{3} \sim x_{1} \succ x_{4}$, as it fails to discriminate $x_{1}$ and $x_{3}$ and underperforms the proposed CSM and the other two methods in both MD and MAD. 
Table 8. Performance comparisons for Example 3

\begin{tabular}{|c|c|c|c|c|c|}
\hline Method & $W^{*}$ & Ranking & $F C R$ & MD & MAD \\
\hline CSM (This article) & $(0.0884,0.1615,0.1581,0.2365,0.2185,0.1370)^{T}$ & $x_{4} \succ x_{5} \succ x_{2} \succ x_{3} \succ x_{6} \succ x_{1}$ & 0.0728 & 0.7487 & 0.1802 \\
\hline EM [40] & $(0.0896,0.1671,0.1594,0.2355,0.2225,0.1258)^{T}$ & $x_{4} \succ x_{5} \succ x_{2} \succ x_{3} \succ x_{6} \succ x_{1}$ & 0.0729 & 0.6047 & 0.1826 \\
\hline NRAM [32] & $(0.1204,0.1681,0.1648,0.2000,0.1931,0.1537)^{T}$ & $x_{4} \succ x_{5} \succ x_{2} \succ x_{3} \succ x_{6} \succ x_{1}$ & 0.1014 & 1.3993 & 0.2345 \\
\hline GPM [41] & $(0.1091,0.1636,0.1091,0.2545,0.2545,0.1091)^{T}$ & $x_{4} \approx x_{5} \succ x_{2} \succ x_{3} \approx x_{6} \approx x_{1}$ & 0.1033 & 1.7849 & 0.2999 \\
\hline LSM [17] & $(0.0978,0.1765,0.1591,0.2263,0.2220,0.1183)^{T}$ & $x_{4} \succ x_{5} \succ x_{2} \succ x_{3} \succ x_{6} \succ x_{1}$ & 0.0778 & 0.6848 & 0.1987 \\
\hline LLSM [38] & $(0.0878,0.1599,0.1551,0.2464,0.2208,0.1301)^{T}$ & $x_{4} \succ x_{5} \succ x_{2} \succ x_{3} \succ x_{6} \succ x_{1}$ & 0.0719 & 0.6037 & 0.1874 \\
\hline
\end{tabular}

Table 9

Performance comparisons for Example 4

\begin{tabular}{|c|c|c|c|c|}
\hline Methods & $W^{*}$ & Ranking & MD & MAD \\
\hline CSM(This article) & $(0.1954,0.4386,0.2316,0.1344)^{T}$ & $x_{2} \succ x_{3} \succ x_{1} \succ x_{4}$ & 0.7520 & 0.1672 \\
\hline LSM[17] & $(0.1822,0.4611,0.2160,0.1408)^{T}$ & $x_{2} \succ x_{3} \succ x_{1} \succ x_{4}$ & 0.9909 & 0.1946 \\
\hline GPM [41] & $(0.2000,0.4667,0.2000,0.1333)^{T}$ & $x_{2} \succ x_{3} \approx x_{1} \succ x_{4}$ & 1.0411 & 0.1999 \\
\hline LLSM[38] & $(0.1864,0.4587,0.2274,0.1275)^{T}$ & $x_{2} \succ x_{3} \succ x_{1} \succ x_{4}$ & 0.8154 & 0.1825 \\
\hline
\end{tabular}




\section{Concluding remarks}

This paper proposes a chi-square method to handle decision problems with incomplete reciprocal preference relations and develops a convergent iterative algorithm to determine a priority vector. An adapted acceptable consistency ratio is employed to judge whether an incomplete reciprocal preference relation is acceptably consistent. If its consistency is not acceptable, an algorithm is put forward to repair it until its consistency reaches Saaty's suggested threshold. This extended CSM not only improves the consistency level but also aims to preserve the initial preference information as much as possible.

Four numerical examples are examined to illustrate how to apply the proposed CSM and its effectiveness. Comparative studies with existing methods reveal the following features of the proposed CSM:

(1) In contrast to LSM, GPM and LLSM where DM's weights are not considered, the proposed CSM allows the analyst to assign proper weights to different experts to reflect their varying influences in GDM problems.

(2) By setting $h_{1}=1$ and $h_{k}=0$ for $k=2, \ldots, m$, CSM can be conveniently applied to derive a priority vector from a single incomplete reciprocal preference relation. This implies that the proposed CSM model can be employed to handle both group and individual decision problems.

(3) By setting $\delta_{i j}=1$, for all $i, j \in N$, CSM can be utilized to derive a priority vector from complete reciprocal preference relations. This indicates that it can be flexibly used to handle decision problems with both complete and incomplete reciprocal preference relations.

(4) Numerical experiments demonstrate that CSM often outperforms the other methods such as EM, GPM, LSM, LLSM, and NRAM in terms of FCR, MD, and MAD when handling incomplete reciprocal preference relations.

(5) As illustrated in Example 2, CSM tends to have better rank preservation capability and discrimination power.

Current research establishes CSM as a viable and effective tool to handle decision 
problems with incomplete reciprocal preference relations. In reality, DMs may provide their preference judgment in different formats of preference relations. As a worthy future research topic, it would be interesting to explore how the CSM framework can be extended to tackle other types of decision inputs such as incomplete intuitionistic fuzzy preference relations [29], incomplete linguistic preference relations $[8,25]$ and related consensus problems $[6,30,31]$.

\section{Acknowledgments}

The authors are very grateful to the Associate Editor and the two anonymous reviewers for their constructive comments and suggestions that have helped to improve the quality and presentation of this paper. Yejun $\mathrm{Xu}$ would like to acknowledge the financial support of National Natural Science Foundation of China (NSFC) Grants (No. 71101043, 71471056 and 71433003), the Fundamental Research Funds for the Central Universities (No. 2014B09214), Program for Excellent Talents in Hohai University. Kevin W. Li would like to acknowledge the financial support of a Natural Sciences and Engineering Research Council of Canada (NSERC) Discovery Grant, and NSFC Grants (No. 71272129 and 71271188).

\section{References}

[1] S. Alonso, F. Cabrerizo, F. Chiclana, F. Herrera, E. Herrera-Viedma, Group decision making with incomplete fuzzy linguistic preference relations, International Journal of Intelligent Systems, 24 (2009) 201-222.

[2] S. Alonso, F. Chiclana, F. Herrera, E. Herrera-Viedma, A learning procedure to estimate missing values in fuzzy preference relations based on additive consistency, in: V. Torra, Y. Narukawa (Eds.) MDAI, 2004, LNAI 3131, Springer-Verlag, 2004, pp. 227-238.

[3] S. Alonso, F. Chiclana, F. Herrera, E. Herrera-Viedma, A consistency-based procedure to estimate missing pairwise preference values, International Journal of Intelligent Systems, 23 (2008) 155-175.

[4] S. Alonso, E. Herrera-Viedma, F. Chiclana, F. Herrera, Individual and social strategies to deal with ignorance situations in multi-person decision making, International Journal of Information Technology \& Decision Making, 8 (2009) 313-333.

[5] S. Alonso, E. Herrera-Viedma, F. Chiclana, F. Herrera, A web based consensus support system for group decision making problems and incomplete preferences, Information Sciences, 180 (2010) 4477-4495.

[6] S. Alonso, I.J. Perez, F.J. Cabrerizo, E. Herrera-Viedma, A linguistic consensus model for Web 2.0 communities, Applied Soft Computing, 13 (2013) 149-157.

[7] E. Blankmeyer, Approaches to consistency adjustment, Journal of Optimization Theory and Applications, 54 (1987) 479-488. 
[8] F. Cabrerizo, I. Pérez, E. Herrera-Viedma, Managing the consensus in group decision making in an unbalanced fuzzy linguistic context with incomplete information, Knowledge-Based Systems, 23 (2010) 169-181.

[9] S.M. Chen, C.S. H., T.E. Lin, Group decision making systems using group recommendations based on interval fuzzy preference relations and consistency matrices, Information Sciences, 298 (2015) 555-567.

[10] S.M. Chen, T.E. Lin, L.W. Lee, Group decision making using incomplete fuzzy preference relations based on the additive consistency and the order consistency, Information Sciences, 259 (2014) 1-15.

[11] F. Chiclana, E. Herrera-Viedma, S. Alonso, A note on two methods for estimating missing pairwise preference values, IEEE Transactions on Systems Man and Cybernetics-Part B: Cybernetics, 39 (2009) 1628-1633.

[12] F. Chiclana, E. Herrera-Viedma, S. Alonso, F. Herrera, Cardinal consistency of reciprocal preference relations: a characterization of multiplicative transitivity, IEEE Transactions on Fuzzy Systems, 17 (2009) 14-23.

[13] F. Chiclana, F. Herrera, E. Herrera-Viedma, Integrating three representation models in fuzzy multipurpose decision making based on fuzzy preference relations, Fuzzy Sets and Systems, 97 (1998) $33-48$.

[14] Y.C. Dong, X. Chen, F. Herrera, Minimizing adjusted simple terms in the consensus reaching process with hesitant linguistic assessments in group decision making, Information Sciences, 297 (2015) 95-117.

[15] M. Fedrizzi, S. Giove, Incomplete pairwise comparison and consistency optimization, European Journal of Operational Research, 183 (2007) 303-313.

[16] J.C. Fodor, M.R. Roubens, Fuzzy preference modelling and multicriteria decision support, Springer, 1994.

[17] Z.W. Gong, Least-square method to priority of the fuzzy preference relations with incomplete information, International Journal of Approximate Reasoning, 47 (2008) 258-264.

[18] F. Herrera, E. Herrera-Viedma, J.L. Verdegay, A sequential selection process in group decision making with a linguistic assessment approach, Information Sciences, 85 (1995) 223-239.

[19] R.E. Jensen, Comparison of eigenvector, least squares, chi square and logarithmic least square methods of scaling a reciprocal matrix, in, Turinity University, Working Paper, 1983.

[20] Y.P. Jiang, Z.P. Fan, An approach to group decision making based on incomplete fuzzy preference relations, International Journal of Uncertainty, Fuzziness and Knowledge-Based Systems, 16 (2008) 83-94.

[21] J. Kacprzyk, Group decision making with fuzzy majority, Fuzzy Sets and Systems, 18 (1986) 105-118.

[22] J. Ma, Z.P. Fan, Y.P. Jiang, J.Y. Mao, L. Ma, A method for repairing the inconsistency of fuzzy preference relations, Fuzzy Sets and Systems, 157 (2006) 20-33.

[23] S.A. Orlovsky, Decision-making with a fuzzy preference relation, Fuzzy Sets and Systems, 1 (1978) 155-167.

[24] W. Pedrycz, P. Ekel, R. Parreiras, Fuzzy Multicriteria Decision-Making: Models, Methods and Applications, Wiley, 2011.

[25] C. Porcel, E. Herrera-Viedma, Dealing with incomplete information in a fuzzy linguistic recommender system to disseminate information in university digital libraries, Knowledge-Based 
Systems, 23 (2010) 32-39.

[26] T.L. Saaty, The Analytic Hierarchy Process, in, McGraw-Hill Company, New York, 1980.

[27] T. Tanino, Fuzzy preference orderings in group decision making, Fuzzy Sets and Systems, 12 (1984) 117-131.

[28] Y.M. Wang, G.W. Fu, The chi-Square priority method of comparison matrix, Journal of Decision and Hierarchy Analytic Process, 2 (1992) 19-27.

[29] J. Wu, F. Chiclana, Multiplicative consistency of intuitionistic reciprocal preference relations and its application to missing values estimation and consensus building, Knowledge-Based Systems, 71 (2014) 187-200.

[30] J. Wu, F. Chiclana, A social network analysis trust consensus based approach to group decision-making problems with interval-valued fuzzy reciprocal preference relations, Knowledge-Based Systems, 59 (2014) 97-107.

[31] J. Wu, F. Chiclana, Visual information feedback mechanism and attitudinal prioritisation method for group decision making with triangular fuzzy complementary preference relations, Information Sciences, 279 (2014) 716-734.

[32] Y.J. Xu, Q.L. Da, Methods for priority of incomplete complementary judgement matrices, Systems Engineering and Electronics, 31 (2009) 95-99.

[33] Y.J. Xu, Q.L. Da, H.M. Wang, A note on group decision-making procedure based on incomplete reciprocal relations, Soft Computing, 15 (2011) 1289-1300.

[34] Y.J. Xu, J.N.D. Gupta, H.M. Wang, The ordinal consistency of an incomplete reciprocal preference relation, Fuzzy Sets and Systems, 246 (2014) 62-77.

[35] Y.J. Xu, K.W. Li, H.M. Wang, Distance-based consensus models for fuzzy and multiplicative preference relations, Information Sciences, 253 (2013) 56-73.

[36] Y.J. Xu, K.W. Li, H.M. Wang, Incomplete interval fuzzy preference relations and their applications, Computers \& Industrial Engineering, 67 (2014) 93-103.

[37] Y.J. Xu, F. Ma, F.F. Tao, H.M. Wang, Some methods to deal with unacceptable incomplete 2-tuple fuzzy linguistic preference relations in group decision making, Knowledge-Based Systems, 56 (2014) 179-190.

[38] Y.J. Xu, R. Patnayakuni, H.M. Wang, Logarithmic least squares method to priority for group decision making with incomplete fuzzy preference relations, Applied Mathematical Modelling, 37 (2013) 2139-2152.

[39] Y.J. Xu, R. Patnayakuni, H.M. Wang, The ordinal consistency of a fuzzy preference relation, Information Sciences, 224 (2013) 152-164.

[40] Y.J. Xu, H.M. Wang, Eigenvector method, consistency test and inconsistency repairing for an incomplete fuzzy preference relation, Applied Mathematical Modelling, 37 (2013) 5171-5183.

[41] Z.S. Xu, Goal programming models for obtaining the priority vector of incomplete fuzzy preference relation, International journal of Approximate Reasoning, 36 (2004) 261-270.

[42] Z.S. Xu, A procedure for decision making based on incomplete fuzzy preference relation, Fuzzy Optimization and Decision Making, 4 (2005) 175-189.

[43] Z.S. Xu, J. Chen, Group decision-making procedure based on incomplete reciprocal relations, Soft Computing, 12 (2008) 515-521.

[44] Y. Zhang, H.X. Ma, Q. Li, B.H. Liu, J. Liu, Conditions of two methods for estimating missing preference information, Information Sciences, 279 (2014) 186-198. 